\title{
Ion channel screening: advances in technologies and analysis
}

\author{
Marzia Martina* \\ Synaptic Therapies and Devices Group, Institute for Biological Sciences, National Research Council of Canada, Ottawa, ON, Canada \\ *Correspondence: marzia.martina@nrc-cnrc.gc.ca
}

This issue provides a comprehensive picture of the most recent technological advances in the field of ion channel screening. Ion channels are proteins that form pores in cell membranes. They are involved in the control of many fundamental physiological processes in various tissues and alterations in their functions give rise to pathological conditions. Ion channels were discovered in the membrane of electrically excitable cells such as neurons, cardiomyocytes, and skeletal muscle fibers and, for decades, drugs modulating cell excitability have been targeted by the pharmaceutical industry. Ion channels also play many roles in non-excitable tissues. Approximately 13\% of marketed drugs act on ion channels (Clare, 2010) and new ion channel screening technologies continue to be developed to discover new drugs.

Patch-clamp is the gold standard technique to record ion channel activity (Hamill et al., 1981). The costs associated with this technique, however, are high as a result of the need for top-quality equipment (e.g., anti-vibrating table, micromanipulator, microscope with IR-CCD camera), highly trained personnel, and low throughput. Over the years, there have been attempts to build devices to increase throughput, notably with automated electrophysiology systems. Such systems have revolutionized ion channel drug discovery by enabling the screening of numerous compounds on many types of ion channels and cellular models. These systems have also been important for safety pharmacology testing of many types of ion channels. These methods, however, need improvements with respect to throughput, quality of the electrophysiological recordings and the physiological cellular models used for drugs and safety testing.

In this issue, Stoelzle et al. (2011) review the development of new automated patch-clamp (APC) systems capable of recording 96 cells in parallel and offering 5,000 data points per day, providing a high throughput per experiment. The development of other important features such as temperature control, the ability to record action potentials, and the testing of drugs on more relevant physiological models improve the quality of results. These APC systems, however, can only be used with suspended cell lines transfected with the ion channels to be studied, thereby not allowing for the study of the processes involved in synaptic communication. Synaptic communication is critical to information processing within neuronal networks - the disruption of which is at the basis of many neurodegenerative diseases - so better understanding of the mechanisms underlying synaptic function requires interrogation of pre- and post-synaptic activity by monitoring/manipulating trans-membrane potential or currents from connected neurons.

Accordingly, there is a need for devices that will be simple to use and lead to higher throughput while providing high quality recordings for both the screening of native neuronal ion channels and the study of neuronal networks. Py et al. (2011) describe a new planar patch-clamp chip in which pipettes are replaced by apertures on a planar surface suitable for neuronal culture. Using synaptically connected snail neurons cultures on these chips, high quality recordings were obtained in both current- and voltageclamp. If successfully translated to mammalian neurons, this new technology will provide an important tool for developing and testing drugs on more relevant physiological disease models (see also Nagarah, 2011).

The development of new technologies has increased the number of drugs that can be identified, synthesized, characterized, screened, and tested for therapeutic efficacy and safety. However, the process of drug discovery is long and expensive. Pharmaceuticals spend $\$ 50$ billion annually in $\mathrm{R} \& \mathrm{D}$ and the average cost to bring a new drug to the market is estimated at $\$ 1.8$ billion (Paul et al., 2010). Most compounds fail in clinical trials, requiring development of new strategies, and methodologies. Kaczorowski et al. (2011) suggest that the profiling of putative lead compounds to identify off-target activities joined with medicinal chemistry research to minimize such activities could save valuable time and money during the preclinical lead optimization phase.

Since ion channels control conduction of electrical activity in the heart, it is crucial to discern if off-target activities of drug candidates include cardiac ion channels. Möller and Witchel (2011) review the most recent methods to screen drug candidates in major cardiac ion channels. These methods include APC using heterologous expression systems and automated action potential recordings from stem-cell derived cardiomyocyte. Polonchuk (2012) evaluates the use of a fully APC system with integrated temperature control - the PatchLiner (Nanion Technologies GmbH, Munich, Germany) - with an ether-á-go-go related gene $\mathrm{K}^{+}$channels assay, in an effort to set a new standard in ion channel research for drug safety testing. For their part, Kaestner and Lipp (2011) describe the advantages of screening cardiac action potentials for drug safety using optical devices to reduce mechanic manipulation of the cells.

Despite the efforts from public and private research to discover new compounds, development of treatment options for many human diseases remains frustratingly slow. It is therefore crucial to continue to seek new therapeutic targets. G protein-coupled inward rectifier $\mathrm{K}^{+}$(GIRK) channels are expressed in the brain, heart and skeletal muscle, and endocrine tissue. GIRK channels are activated via $G$ protein-coupled receptors and regulate the electrical activity of neurons, cardiomyocytes, and $\beta$-pancreatic cells, making them an important target for new drug discovery for treatment of neuropathic pain, drug addition, and cardiac arrhythmias (atrial fibrillation). Walsh (2011) describes the development of a screening assay which uses pituitary and cardiac cell lines expressing GIRK channels combined with patch-clamp and imaging techniques to expand on the limited pharmacology of these channels. Raphemot et al. (2011) describe the use of a thallium (see Van der Heyden, 2012 for commentary) flux-based fluorescence assay to screen a 
Kir1.1 inhibitor library for antagonists of GIRK. Their finding of a new compound, VU573, could be useful to investigate the function and pharmacology of these channels.

Given the importance of identifying new therapeutic targets, the work of Sitzia et al. (2011) on $\alpha 7$ nicotinic receptors - involved in schizophrenia and other diseases involving cognitive impairment as well as in neuroprotection - and their finding of the role played

\section{REFERENCES}

Clare, J. J. (2010). Targeting ion channels for drug discovery. Discov. Med. 9, 253-260.

Hamill, O. P., Marty, A., Neher, E., Sakmann, B., and Sigworth, F.J. (1981). Improved patch-clamp techniques for high-resolution current recording from cells and cell-free membrane patches. Pflügers Arch. 391, 85-100.

Kaczorowski, G. J., Garcia, M. L., Bode, J., Hess, S. D., and Patel, U. A. (2011). The importance of being profiled: improving drug candidate safety and efficacy using ion channel profiling. Front. Pharmacol. 2:78. doi: 10.3389/ fphar.2011.00078

Kaestner,L., and Lipp, P. (2011). Screening action potentials: the power of light. Front. Pharmacol. 2:42. doi: 10.3389/ fphar.2011.00042

Möller, C., and Witchel, H. (2011). Automated electrophysiology makes the pace for cardiac ion channel safety screening. Front. Pharmacol.2:73. doi: 10.3389/fphar.2011.00073
Nagarah, J. M. (2011). Neurons on a chip - toward high throughput network and pharmacology investigations. Front. Pharmacol.2:74. doi: 10.33989/ fphar.2011.00074

Paul, S. M., Mytelka, D. S., Dunwiddie, C. T., Persinger, C. C., Munos, B. H., Lindborg, S. R., and Schacht, A. L. (2010). How to improve $R \& D$ productivity: the pharmaceutical industry's grand challenge. Nat. Rev. Drug Discov. 9, 203-214.

Polonchuk, L. (2012). Toward a new gold standard for early safety: automated temperature-controlled hERG test on the PatchLiner ${ }^{\circledR}$. Front. Pharmacol. 2:3. doi: 10.3389/fphar.2012.00003

Py, C., Martina, M., Diaz-Quijada, G. A., Luk, C. C., Martinez, D., Denhoff, M. D., Charrier,A., Comas, T., Monette, R., Krantis, A., Syed, N. I., and Mealing, G. A. R. (2011). From understanding cellular function to novel drug discovery: the role of planar patch-clamp array chip technology. Front. Pharmacol. 2:51. doi: 10.3389/fphar.2011.00051

by temperature variations in the modulation of these receptors is an appropriate way of concluding this overview of recent advances in ion channel screening.

The huge developments in the field of ion channel screening technologies described in this issue clearly indicate that the future is pointed toward the screening of drugs in more physiological relevant cellular models and networks.

Raphemot, R., Lonergan, D. F., Nguyen, T T., Utley, T., Lewis, L. L., Kadakia, R. Weaver, C. D., Gogliotti, R., Hopkins, C., Lindsley, C. W., and Denton, J. S. (2011). Discovery, characterization, and structure-activity relationships of an inhibitor of inward rectifier potassium (Kir) channels with preference for Kir2.3, Kir3.X, and Kir7.1. Front. Pharmacol. 2:75. doi: 10.3389/ fphar.2011.00075

Sitzia, F., Brown, J. T., Randall, A. D., and Dunlop, J. (2011). Voltage- and temperature-dependent allosteric modulation of $\alpha 7$ nicotinic receptors by PNU120596. Front. Pharmacol. 2:81. doi: 10.3389/fphar.2011.00081

Stoelzle, S., Obergrussberger, A., Brüggemann, A., Haarmann, C., George, M., Kettenhofen, R., and Fertig, N. (2011). State-of-the-art automated patch clamp devices: heat activation, action potentials, and high throughput in ion channel screening. Front. Pharmacol. 2:76. doi: 10.3389/ fphar.2011.00076
Van der Heyden, M.A. G. (2012). Finding inward rectifier channel inhibitors: why and how? Front. Pharmacol. 2:95 doi: 10.3389/fphar.2011.00095

Walsh, K. B. (2011). Targeting GIRK channels for the development of new therapeutic agents. Front. Pharmacol. 2:64. doi: 10.3389/fphar.2011.00064

Received: 16 April 2012; accepted: 17 April 2012; published online: 07 May 2012.

Citation: Martina M (2012) Ion channel screening: advances in technologies and analysis. Front. Pharmacol. 3:86. doi: 10.3389/fphar.2012.00086

This article was submitted to Frontiers in Pharmacology of Ion Channels and Channelopathies, a specialty of Frontiers in Pharmacology.

Copyright (c) 2012 Martina. This is an open-access article distributed under the terms of the Creative Commons Attribution Non Commercial License, which permits non-commercial use, distribution, and reproduction in other forums, provided the original authors and source are credited. 